\title{
Clinical Factors Associated with Long Fluoroscopy Time in Percutaneous Coronary Interventions to the Culprit Lesion of Non-ST-Segment Elevation Myocardial Infarction
}

\author{
Shun Ishibashi, ${ }^{1}$ MD, Kenichi Sakakura, ${ }^{1}$ MD, Satoshi Asada, ${ }^{1}$ MD, Yousuke Taniguchi, ${ }^{1}$ MD, \\ Kei Yamamoto, ${ }^{1}$ MD, Takunori Tsukui, ${ }^{1}$ MD, Masaru Seguchi, ${ }^{1}$ MD, Hiroyuki Jinnouchi, ${ }^{1}$ MD, \\ Hiroshi Wada, ${ }^{1}$ MD and Hideo Fujita, ${ }^{1}$ MD
}

\begin{abstract}
Summary
The clinical outcomes of patients with non-ST-segment elevation myocardial infarction (NSTEMI) were comparable or even worse than those with ST-segment elevation myocardial infarction (STEMI). Although successful percutaneous coronary intervention (PCI) to the culprit lesions of NSTEMI would improve the clinical outcomes, some PCI require long fluoroscopy time, reflecting the difficulty of PCI. This study aims to find clinical factors associated with long fluoroscopy time in PCI to the culprit lesion of NSTEMI. We included 374 patients and divided those into the conventional fluoroscopy time $(n=302)$ and long fluoroscopy time $(n=72)$ groups according to the quintiles of fluoroscopy time. Clinical and angiographic parameters were compared between the two groups. Calcification and tortuosity were significantly more severe in the long fluoroscopy time group than in the conventional fluoroscopy time group. The prevalence of previous coronary artery bypass grafting $(\mathrm{CABG})$ and bifurcation lesions was significantly greater in the long fluoroscopy time group than in the conventional fluoroscopy time group. In the multivariate stepwise logistic regression analysis, previous $\mathrm{CABG}$ (odds ratio [OR], 3.368; 95\% confidence interval [CI], 1.407-8.064; $P=0.006$ ), bifurcation lesion (OR, 2.407; 95\% CI, 1.285-4.506; $P=0.006$ ), excessive tortuosity (versus mild to moderate tortuosity; OR, 4.095; 95\% CI, 1.159-14.476; $P=0.029$ ), and moderate to severe calcification (versus none to mild; OR, 5.792; 95\% CI, 3.254-10.310; $P<0.001$ ) were significantly associated with long fluoroscopy time. In conclusion, previous $\mathrm{CABG}$, bifurcation, excessive tortuosity, and moderate to severe calcification were associated with long fluoroscopy time. Our study provided a reference for PCI operators to identify the difficulties in PCI to the culprit lesion of NSTEMI.
\end{abstract}

(Int Heart J 2021; 62: 282-289)

Key words: Acute myocardial infarction, Calcification, Tortuosity, Bifurcation

A cute myocardial infarction (AMI) remains the leading cause of death in developed countries ${ }^{1}$ and is classified into ST-segment elevation myocardial infarction (STEMI) and non-ST-segment elevation myocardial infarction (NSTEMI), because the initial treatment strategy is different between STEMI and NSTEMI. ${ }^{2,3)}$ Unlike STEMI, NSTEMI may not require emergent revascularization in clinical practice. ${ }^{3)}$ However, the clinical outcomes of patients with NSTEMI were comparable or even worse than those of patients with STEMI, ${ }^{4,5)}$ partly because the severity of NSTEMI widely varied from no myocardial injury with slight elevation of high-sensitivity troponin to cardiogenic shock caused by left main disease. ${ }^{6,7)}$ Therefore, it would be important for patients with NSTEMI to select an appropriate treatment strategy for each scenario.

According to the current guidelines of the Japanese Circulation Society, ${ }^{8)}$ early invasive treatment is recom- mended for high-risk patients with NSTEMI. Successful percutaneous coronary intervention (PCI) to the culprit lesion of NSTEMI would improve the clinical outcomes of patients with NSTEMI.9) Although achieving successful reperfusion in PCI to the culprit lesion of NSTEMI is technically easier as compared to PCI to chronic total occlusion (CTO) ${ }^{10)}$ sometimes, achieving reperfusion even in PCI to the culprit lesion of NSTEMI is difficult. ${ }^{11)}$ Such difficulty in PCI may affect the clinical outcomes in patients with NSTEMI. Since the fluoroscopy time in PCI is considered to reflect difficulty in $\mathrm{PCI},{ }^{12,13)}$ long fluoroscopy time in PCI to the culprit lesion of NSTEMI would mean that such PCI was technically difficult. This study aims to find clinical factors associated with long fluoroscopy time in PCI to the culprit lesion of NSTEMI.

From the ${ }^{1}$ Division of Cardiovascular Medicine, Saitama Medical Center, Jichi Medical University, Saitama, Japan.

Address for correspondence: Kenichi Sakakura, MD, Division of Cardiovascular Medicine, Saitama Medical Center, Jichi Medical University, 1-847 Amanuma, Omiya-ku, Saitama, Saitama 330-8503, Japan. E-mail: ksakakura@jichi.ac.jp

Received for publication September 15, 2020. Revised and accepted November 4, 2020.

Released in advance online on J-STAGE March 17, 2021.

doi: 10.1536/ihj.20-634

All rights reserved by the International Heart Journal Association. 


\section{Methods}

Study lesions: We identified consecutive patients with AMI in our institution from January 2015 to December 2018. The inclusion criteria were as follows: (1) patients with NSTEMI and (2) patients who underwent PCI to the culprit lesion of NSTEMI. The exclusion criteria were as follows: (1) patients who were treated with medical therapy alone, (2) patients who underwent coronary artery bypass graft surgery to the culprit lesion, and (3) patients who underwent PCI to the non-culprit lesion and the culprit lesion of NSTEMI simultaneously. The final study population was divided into the long fluoroscopy time and conventional fluoroscopy time groups according to the quintiles of fluoroscopy time. The long fluoroscopy time group was defined as the longest quintiles of fluoroscopy time (Q5), whereas the conventional fluoroscopy time group was defined as the other quintiles of fluoroscopy time (Q1-Q4). This study was approved by the Institutional Review Board of Saitama Medical Center, Jichi Medical University (S20-051), and written informed consent was waived because of the retrospective study design. PCI to the culprit of NSTEMI: PCI procedures were performed on a biplane fluoroscopy system (Artis zee BC and Artis zee BA, Siemens, Munich, Germany), with three magnetic fields $(16,20$, and $25 \mathrm{~cm}$ for Artis zee BC; 16 , 22 , and $32 \mathrm{~cm}$ for Artis zee BA) and a standard image acquisition program at 15 frames per second during cine acquisition and 7.5 frames per second during fluoroscopy. ${ }^{14)}$ The choice of PCI devices, such as guidewire, balloon, thrombectomy device, rotational atherectomy device, and stent, was left at the discretion of interventional cardiologists at our cardiology center. For bifurcation lesions, we rarely considered two-stent strategy. Single-stent implantation followed by kissing balloon techniques (KBT) or proximal optimization techniques (POT) was performed in most bifurcation cases. ${ }^{15,16)}$ IVUS was routinely used for almost all lesions.

Definition: AMI was defined based on universal definition of myocardial infarction. ${ }^{17)}$ NSTEMI was diagnosed as AMI without persistent ST elevation on electrocardiogram. ${ }^{18)}$ Hypertension was defined as medical treatment for hypertension and/or a history of hypertension before admission. ${ }^{19)}$ Dyslipidemia was defined as a total cholesterol level $\geq 220 \mathrm{mg} / \mathrm{dL}$ or low-density lipoprotein cholesterol level $\geq 140 \mathrm{mg} / \mathrm{dL}$ or medical treatment for dyslipidemia or a history of dyslipidemia. ${ }^{19,20)}$ Diabetes mellitus was defined as a hemoglobin A1c level $\geq 6.5 \%$ (as NGSP value) or medical treatment for diabetes mellitus or a history of diabetes mellitus. ${ }^{19,20)}$ We calculated the estimated glomerular filtration rate (eGFR) from the serum creatinine level, age, weight, and gender using the following formula: $\mathrm{eGFR}=194 \times \mathrm{Cr}-1.094 \times \mathrm{x}$ age-0.287 for male and $\mathrm{eGFR}=194 \times \mathrm{Cr}-1.094 \times$ age-0.287 $\times 0.739$ for female. $^{21)}$

Angiographic analysis: Quantitative coronary angiography parameters were measured using a cardiovascular angiography analysis system (QAngio XA 7.3, MEDIS Imaging Systems, Leiden, Netherlands). The definition of lesion characteristics, including lesion length, eccentricity, tortuosity, lesion angle, ostial lesion, and bifurcation le- sion, has been previously described. ${ }^{22)}$ As known, bifurcation lesion is defined as the side branch requiring wire protection from the culprit lesion. ${ }^{22)}$ In an original ACC/ AHA definition, tortuosity was defined as follows: stenoses distal to two bends were defined as moderately tortuous, and those distal to three or more bends were defined as excessive tortuosity. ${ }^{22)}$ However, since the above definition of tortuosity has poor objectivity, we additionally defined tortuosity as follows: " $\leq 1$ " moderate to excessive bend $\left(<120^{\circ}\right)$, mild tortuosity; " 2 " moderate to excessive bend $\left(<120^{\circ}\right)$, moderate tortuosity; and " $\geq 3$ " moderate to excessive bend $\left(<120^{\circ}\right)$, excessive tortuosity. ${ }^{23)}$ Calcification was identified as readily apparent radiopacities within the vascular wall at the site of the stenosis and was classified as none/mild, moderate (radiopacities noted only during the cardiac cycle before contrast injection), and severe (radiopacities noted without cardiac motion before contrast injection generally compromising both sides of the arterial lumen). ${ }^{24}$

Statistical analysis: Data are shown as the mean \pm SD or percentage. Categorical variables are presented as numbers (percentage) and were compared using the Pearson $\chi^{2}$ test. Continuous variables were compared between the groups using the unpaired Student's test. Univariate and multivariate logistic regression analyses were performed to find the association between the clinical variables and long fluoroscopy time. In this model, long fluoroscopy time (Q 5) was used as the dependent variable. In the multivariate stepwise logistic regression model, the selection of independent variables was derived from the results of univariate logistic regression analysis $(P<0.05$ in univariate analysis). However, variables with missing values were not included in the model. The multivariate logistic regression analysis with likelihood ratio statistical criteria using backward elimination method was performed. The odds ratio (OR) and 95\% confidence interval (CI) were also calculated. A $P$ value $<0.05$ was considered statistically significant. We analyzed all data using SPSS ver. 24 for Windows (SPSS, Inc., Chicago, Illinois).

\section{Results}

During the study period, we had a total of 465 patients with NSTEMI in our medical center. We excluded 91 patients according to the exclusion criteria. Our final study population was 374 patients, which was then divided into the long fluoroscopy time $(n=72)$ and conventional fluoroscopy time $(n=302)$ groups. The study flowchart is shown in the Figure.

The comparison of clinical, lesion, and procedural characteristics is shown in Table I. Calcification was significantly more severe in the long fluoroscopy time group than in the conventional fluoroscopy time group $(P<$ 0.001). Similarly, there were significant differences in the history of previous coronary artery bypass grafting (CABG), number of narrowed coronary arteries, left main trunk lesion, bifurcation lesion, and tortuosity between the two groups. The univariate logistic regression models to find the factors associated with long fluoroscopy time are shown in Table II. Complex lesion characteristics, such as calcification, tortuosity, left main trunk lesion, and bifur- 


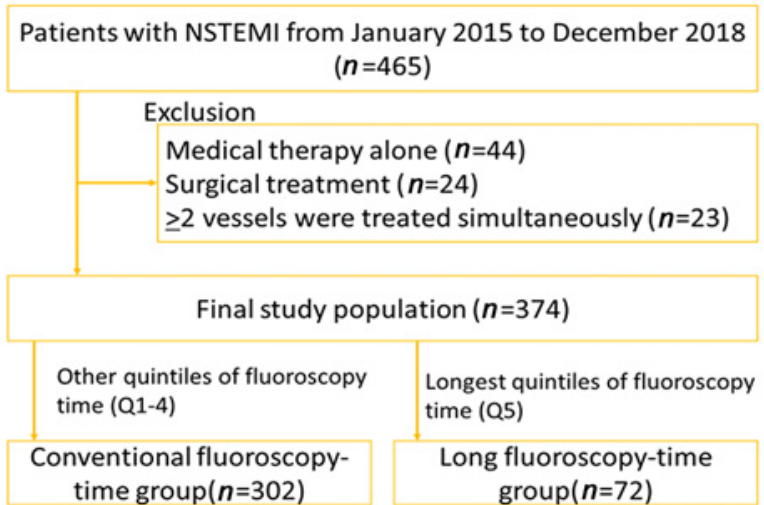

Figure. The study flowchart. NSTEMI indicates non-ST-segment elevation myocardial infarction.

cation lesion, were significantly associated with long fluoroscopy time. Furthermore, some procedural characteristics, such as the guiding catheter size, rotational atherectomy use, intra-aortic balloon pumping support, and venoarterial extracorporeal membrane oxygenation, were also associated with long fluoroscopy time.

The multivariate stepwise logistic regression analysis to find the factors associated with long fluoroscopy time is shown in Table III. The stepwise model included the following variables: $\mathrm{CABG}$ history, triple-vessel disease (versus $\leq 2$-vessel diseases), left main trunk lesion, bifurcation lesion, excessive tortuosity (versus mild to moderate tortuosity), moderate to severe calcification (versus none to mild), transradial coronary intervention (versus TFI or TBI), and rotational atherectomy use. Previous CABG (OR, 3.368; 95\% CI, 1.407-8.064; $P=0.006$ ), bifurcation lesion (OR, 2.407; 95\% CI, 1.285-4.506; $P=$ 0.006 ), excessive tortuosity (versus mild to moderate tortuosity; OR, 4.095; 95\% CI, 1.159-14.476; $P=0.029)$, and moderate to severe calcification (versus none to mild; OR, 5.792; 95\% CI, 3.254-10.310; $P<0.001$ ) were significantly associated with long fluoroscopy time in this final model.

\section{Discussion}

We included 374 patients who underwent PCI to the culprit lesion of NSTEMI and divided those into the conventional fluoroscopy time $(n=302)$ and long fluoroscopy time $(n=72)$ groups according to the quintiles of fluoroscopy time. Calcification and tortuosity were significantly more severe in the long fluoroscopy time group than in the conventional fluoroscopy time group. The prevalence of previous $\mathrm{CABG}$ and bifurcation lesions were significantly greater in the long fluoroscopy time group than in the conventional fluoroscopy time group. In the multivariate stepwise logistic regression analysis, previous $\mathrm{CABG}$, bifurcation lesion, excessive tortuosity, and moderate to severe calcification were significantly associated with long fluoroscopy time.

In the present study, a history of previous CABG was significantly associated with long fluoroscopy time, whereas the graft lesion as the culprit of NSTEMI was not associated with long fluoroscopy time. Thus, PCI to the native coronary artery in patients with previous CABG would require long fluoroscopy time. In general, native coronary arteries in patients with previous $\mathrm{CABG}$ progress to severe stenosis or occlusion in long term, suggesting complex atherosclerotic lesions in the native coronary arteries. ${ }^{25)}$ Such lesion complexity in native coronary arteries might be the reason for longer fluoroscopy time in patients with previous $\mathrm{CABG}$.

Bifurcation lesions were significantly associated with long fluoroscopy time in the present study. Although we rarely considered two-stent strategy, bifurcation lesions required additional techniques such as KBT or POT, ${ }^{15,16)}$ which would consume additional fluoroscopy time. Guidewire protection would also require additional fluoroscopy time. Moreover, the recrossing of the guidewire via stent struts was sometimes difficult, even if we used a double-lumen microcatheter such as crusade (Kaneka, Osaka, Japan). ${ }^{26)}$ Those additional procedures would be associated with longer fluoroscopy time in bifurcation lesions.

Excessive tortuosity was significantly associated with long fluoroscopy time in the present study. Several groups reported that excessive tortuosity could be the reason for unsuccessful stent delivery. ${ }^{27,28)}$ Our group also reported that excessive tortuosity was associated with long fluoroscopy time in primary PCI for STEMI. ${ }^{23)}$ Furthermore, Wilson, et al. reported that the cause of unsuccessful PCI in CTO lesions was not calcification but was within-CTO tortuosity. ${ }^{29)}$ Therefore, in the present study, excessively tortuous lesions would require additional fluoroscopy time to deliver stents or other devices. Moderate to severe calcification was significantly associated with long fluoroscopy time in the present study. Calcification is also known to be an important factor that makes device delivery difficult $^{27)}$ and a factor associated with unsuccessful PCI. ${ }^{30,31)}$ Severe calcification may require a variety of devices such as rotational atherectomy and cutting balloons, which would increase fluoroscopy time. ${ }^{32)}$ In fact, rotational atherectomy was used in approximately $10 \%$ of our study population.

The clinical implications of the present study should be noted. Owing to the advancement of early invasive strategy, ${ }^{9)}$ most PCI to the culprit lesions of NSTEMI might be performed by junior PCI operators as emergent or urgent $\mathrm{PCI}{ }^{33)}$ Unlike primary PCI, more than half of PCI lesions in this study showed initial TIMI flow grade 3 , which would be the goal of primary PCI. ${ }^{34)}$ Therefore, the goal of PCI in patients with NSTEM is not achieving TIMI flow grade 3 but achieving complete revascularization to the culprit lesion of NSTEMI. In other words, the technical quality of each PCI is more important in the culprit lesions of NSTEMI than in the culprit lesions of STEMI. If the culprit lesion of NSTEMI shows complex features, such as bifurcation, excessive tortuosity, and moderate to severe calcification, those lesions may be too difficult for junior operators without the backup of senior operators. Performing emergent coronary angiography and postponing emergent PCI would be possible in most NSTEMI cases, as long as TIMI flow grade 3 was maintained. The present study provided a reference to stratify 
Table I. Comparison of Patient Characteristics Between the Conventional Fluoroscopy Time and Long Fluoroscopy Time Groups

\begin{tabular}{|c|c|c|c|}
\hline & $\begin{array}{l}\text { Conventional fluoroscopy time group } \\
\qquad n=302\end{array}$ & $\begin{array}{l}\text { Long fluoroscopy time group } \\
\qquad n=72\end{array}$ & $P$ value \\
\hline Age, years & $71.6 \pm 11.0$ & $73.1 \pm 10.4$ & 0.233 \\
\hline Male, $n(\%)$ & $223(73.8)$ & $53(73.6)$ & 0.968 \\
\hline \multicolumn{4}{|l|}{ Physical examination } \\
\hline Height $(\mathrm{cm})$ & $161.3 \pm 11.2(n=298)$ & $160.1 \pm 8.7$ & 0.114 \\
\hline Body weight $(\mathrm{kg})$ & $63.2 \pm 13.8(n=301)$ & $61.2 \pm 11.3$ & 0.273 \\
\hline Systolic blood pressure at admission $(\mathrm{mmHg})$ & $145.1 \pm 30.6(n=294)$ & $147.2 \pm 32.7(n=71)$ & 0.621 \\
\hline Diastolic blood pressure at admission $(\mathrm{mmHg})$ & $81.2 \pm 20.0(n=292)$ & $77.1 \pm 20.3(n=71)$ & 0.121 \\
\hline Heart rate at admission (beat per minute) & $84.0 \pm 21.7(n=296)$ & $80.5 \pm 23.3(n=71)$ & 0.166 \\
\hline \multicolumn{4}{|l|}{ Underlying disease } \\
\hline Hypertension, $n(\%)$ & $262(86.8)$ & $66(91.7)$ & 0.254 \\
\hline Diabetes mellitus, $n(\%)$ & $147 / 300(49.2)$ & $39(54.2)$ & 0.446 \\
\hline Dyslipidemia, $n(\%)$ & $220(74.3)$ & $55(76.4)$ & 0.718 \\
\hline Hemodialysis, $n(\%)$ & $46(15.2)$ & $14(19.4)$ & 0.381 \\
\hline History of previous PCI, $n(\%)$ & $110(36.4)$ & $26(36.1)$ & 0.960 \\
\hline History of previous CABG, $n(\%)$ & $21(7.0)$ & $12(16.7)$ & 0.009 \\
\hline History of previous MI, $n(\%)$ & $81(26.8)$ & $12 / 71(16.9)$ & 0.082 \\
\hline Current smoker, $n(\%)$ & $74 / 301(24.7)$ & $11(15.3)$ & 0.860 \\
\hline \multicolumn{4}{|l|}{ Medication before admission } \\
\hline Aspirin, $n(\%)$ & $163 / 299(54.5)$ & $40 / 71(56.3)$ & 0.781 \\
\hline Thienopyridine, $n(\%)$ & $104 / 299(34.8)$ & $30 / 71(42.3)$ & 0.239 \\
\hline Beta blocker, $n(\%)$ & $117 / 298(39.3)$ & 29/71 (40.8) & 0.806 \\
\hline ACE inhibitor, $\mathrm{ARB}, n(\%)$ & $149 / 298(50.0)$ & $36 / 71(50.7)$ & 0.915 \\
\hline Calcium channel blocker, $n(\%)$ & $127 / 298(42.6)$ & $31 / 71(44.3)$ & 0.800 \\
\hline Statin, $n(\%)$ & $162 / 299(54.2)$ & $41 / 71(57.7)$ & 0.587 \\
\hline Diuretic, $n(\%)$ & $66 / 299(22.1)$ & $9 / 70(12.9)$ & 0.085 \\
\hline Hypoglycemic agents, $n(\%)$ & $88 / 299(29.4)$ & $25 / 71(35.2)$ & 0.342 \\
\hline Insulin, $n(\%)$ & $29 / 299(9.7)$ & $4 / 70(5.7)$ & 0.293 \\
\hline \multicolumn{4}{|l|}{ Laboratory data at admission } \\
\hline Serum creatinine $(\mathrm{mg} / \mathrm{dL})$ & $2.13 \pm 2.83$ & $2.44 \pm 3.19$ & 0.551 \\
\hline Estimated GFR (mL/minute/1.73 $\left.\mathrm{m}^{2}\right)$ & $53.6 \pm 29.3$ & $52.3 \pm 29.7$ & 0.684 \\
\hline Hemoglobin $(\mathrm{g} / \mathrm{dL})$ & $12.8 \pm 2.16$ & $12.6 \pm 2.17$ & 0.651 \\
\hline $\mathrm{BNP}(\mathrm{pg} / \mathrm{mL})$ & $556 \pm 803(n=288)$ & $465 \pm 744(n=71)$ & 0.963 \\
\hline Killip class & & & 0.061 \\
\hline 1 or 2 & $238(78.8)$ & $60(83.3)$ & \\
\hline 3 & $45(14.9)$ & $9(12.5)$ & \\
\hline 4 & $19(6.3)$ & $3(4.2)$ & \\
\hline Cardiac arrest at prehospital or ER, $n(\%)$ & $13(4.3)$ & $2(2.8)$ & 0.553 \\
\hline Shock vital at prehospital or ER, $n(\%)$ & 23/299 (7.7) & $6 / 70(8.6)$ & 0.806 \\
\hline Culprit lesion & & & 0.078 \\
\hline Left main - left anterior descending artery, $n(\%)$ & $137(45.4)$ & $27(38.0)$ & \\
\hline Right coronary artery, $n(\%)$ & $81(26.8)$ & $22(31.0)$ & \\
\hline Left circumflex, $n(\%)$ & $75(24.8)$ & $15(21.1)$ & \\
\hline Graft/other, $n(\%)$ & $4(1.3)$ & $2(2.8)$ & \\
\hline Not determined, $n(\%)$ & $5(1.7)$ & $5(7.0)$ & \\
\hline Number of narrowed coronary arteries & & & 0.012 \\
\hline One-vessel disease, $n(\%)$ & $118(39.1)$ & $22(30.6)$ & \\
\hline Two-vessel disease, $n(\%)$ & $111(36.8)$ & $20(27.8)$ & \\
\hline Three-vessel disease, $n(\%)$ & $73(24.2)$ & $30(41.7)$ & \\
\hline Left main trunk lesion, $n(\%)$ & $32(10.6)$ & $18(25.0)$ & 0.001 \\
\hline Left main trunk bifurcation lesion, $n(\%)$ & $16(5.3)$ & $10(13.9)$ & 0.010 \\
\hline Ostia lesion, $n(\%)$ & $34(11.3)$ & $11(15.3)$ & 0.346 \\
\hline Bifurcation lesion, $n(\%)$ & $66(21.9)$ & $27(37.5)$ & 0.006 \\
\hline Initial TIMI flow grade of culprit & & & 0.107 \\
\hline 0 & $36(11.9)$ & $10(14.1)$ & \\
\hline 1 & $24(7.9)$ & $3(4.2)$ & \\
\hline 2 & $44(14.6)$ & $18(25.4)$ & \\
\hline 3 & $198(65.6)$ & $40(56.3)$ & \\
\hline Lesion length (mm) & $14.0 \pm 10.8$ & $16.2 \pm 12.8$ & 0.340 \\
\hline Reference diameter (mm) & $2.45 \pm 0.67$ & $2.47 \pm 0.80$ & 0.870 \\
\hline Tortuosity, $n(\%)$ & & & 0.005 \\
\hline Mild tortuosity & $251(83.1)$ & $49(68.1)$ & \\
\hline Moderate tortuosity & $44(14.6)$ & $17(23.6)$ & \\
\hline Excessive tortuosity & $7(2.3)$ & $6(8.3)$ & \\
\hline
\end{tabular}


Table I. Comparison of Patient Characteristics Between the Conventional Fluoroscopy Time and Long Fluoroscopy Time Groups (continued)

\begin{tabular}{|c|c|c|c|}
\hline & $\begin{array}{l}\text { Conventional fluoroscopy time group } \\
\qquad n=302\end{array}$ & $\begin{array}{l}\text { Long fluoroscopy time group } \\
\qquad n=72\end{array}$ & $P$ value \\
\hline Eccentricity, $n(\%)$ & & & 0.507 \\
\hline Concentric & $276(91.4)$ & $64(88.9)$ & \\
\hline Eccentric & $26(8.6)$ & $8(11.1)$ & \\
\hline Calcification, $n(\%)$ & & & $<0.001$ \\
\hline None to mild & $246(81.5)$ & $29(40.3)$ & \\
\hline Moderate & $37(12.3)$ & $14(19.4)$ & \\
\hline Severe & $19(6.3)$ & $29(40.3)$ & \\
\hline Occurrence of slow flow in PCI, $n(\%)$ & $8(2.6)$ & $5(6.9)$ & 0.074 \\
\hline Not ad hoc PCI, $n(\%)$ & $112(37.1)$ & $25(34.7)$ & 0.708 \\
\hline Approach site, $n(\%)$ & & & 0.008 \\
\hline Transradial coronary intervention & $184(61.9)$ & $31(43.1)$ & \\
\hline Transfemoral coronary intervention & $15(5.0)$ & $8(11.1)$ & \\
\hline Transbrachial coronary intervention & $100(33.1)$ & $33(45.8)$ & \\
\hline Change of approach site, $n(\%)$ & $10(3.3)$ & $9(12.5)$ & 0.001 \\
\hline PCI procedure, $n(\%)$ & & & 0.342 \\
\hline Plain old balloon angioplasty & $18(6.1)$ & $6(8.6)$ & \\
\hline Aspiration only & $1(0.3)$ & 0 & \\
\hline Drug-coated balloon angioplasty & $29(9.8)$ & $5(7.1)$ & \\
\hline Bare metal stent & $5(1.7)$ & $1(1.4)$ & \\
\hline Drug-eluting stent & $243(81.8)$ & $56(80.0)$ & \\
\hline POBA and aspiration & $1(0.3)$ & 0 & \\
\hline Size of guiding catheter $(\mathrm{Fr})$ & & & $<0.001$ \\
\hline 6 & $176 / 297(59.3)$ & $22 / 70(31.4)$ & \\
\hline 7 & $118 / 297(39.7)$ & $45 / 70(64.3)$ & \\
\hline 8 & $3 / 297(1.0)$ & $3 / 70(4.3)$ & \\
\hline Number of guiding catheters & & & $<0.001$ \\
\hline 1 & $286(94.7)$ & $57(79.2)$ & \\
\hline 2 & $15(5.0)$ & $12(16.7)$ & \\
\hline$\geq 3$ & $1(0.3)$ & $3(4.2)$ & \\
\hline Number of guidewires & & & $<0.001$ \\
\hline 1 & $184(60.9)$ & $16(22.2)$ & \\
\hline 2 & $89(29.5)$ & $23(31.9)$ & \\
\hline$\geq 3$ & $29(9.6)$ & $38(52.8)$ & \\
\hline Number of stents & & & $<0.001$ \\
\hline 1 & $224(90.3)$ & $39(66.1)$ & \\
\hline 2 & $23(9.3)$ & $16(27.1)$ & \\
\hline$\geq 3$ & $1(0.4)$ & $4(6.8)$ & \\
\hline Number of balloons & & & $<0.001$ \\
\hline 1 & $128(42.4)$ & $13(18.1)$ & \\
\hline 2 & $119(39.4)$ & $20(27.8)$ & \\
\hline$\geq 3$ & $47(15.6)$ & $38(52.8)$ & \\
\hline Use of guide extension catheter, $n(\%)$ & $32(10.6)$ & $20(27.8)$ & $<0.001$ \\
\hline Use of microcatheter, $n(\%)$ & $76(25.2)$ & $50(69.4)$ & $<0.001$ \\
\hline Use of aspiration catheter, $n(\%)$ & $10(3.3)$ & $3(4.2)$ & 0.726 \\
\hline Use of rotational coronary atherectomy, $n(\%)$ & $18(6.0)$ & $18(25.0)$ & $<0.001$ \\
\hline IVUS guide, $n(\%)$ & $281(93.0)$ & $70(97.2)$ & 0.185 \\
\hline OCT/OFDI guide, $n(\%)$ & $16(5.3)$ & $1(1.4)$ & 0.152 \\
\hline Temporary pacemaker, $n(\%)$ & $6(2.0)$ & $9(2.4)$ & $<0.001$ \\
\hline Intra-aortic balloon pumping support, $n(\%)$ & $18(4.8)$ & $13(3.5)$ & 0.001 \\
\hline V-A ECMO, $n(\%)$ & $2(0.7)$ & $4(5.6)$ & 0.003 \\
\hline Amount of contrast media $(\mathrm{mL})$ & $112.9 \pm 45.1(n=292)$ & $156.0 \pm 61.2(n=66)$ & $<0.001$ \\
\hline Fluoroscopy time (minutes) & $19.2 \pm 6.8$ & $46.8 \pm 15.7$ & $<0.001$ \\
\hline
\end{tabular}

Data are expressed as the mean \pm SD or number (percentage). A Student's $t$ test was used for normally distributed continuous variables, a Mann-Whitney $U$ test was used for abnormally distributed continuous variables, and a chi-square test was used for categorical variables. PCI indicates percutaneous coronary intervention; CABG, coronary artery bypass grafting; MI, myocardial infarction; ACE, angiotensin-converting enzyme; ARB, angiotensin II receptor blocker; GFR, glomerular filtration rate; BNP, brain natriuretic peptide; ER, emergency room; TIMI, thrombolysis in myocardial infarction; IVUS, intravascular ultrasound; OCT/OFDI, optical coherence tomography/optical frequency domain imaging; and V-A ECMO, veno-arterial extracorporeal membranous oxygenation. 
Table II. Univariate Logistic Regression Analysis to Find Association with Long Fluoroscopy Time

\begin{tabular}{|c|c|c|c|c|c|c|c|}
\hline \multicolumn{4}{|c|}{ Dependent variable: long fluoroscopy time (Q5) } & \multicolumn{4}{|c|}{ Dependent variable: long fluoroscopy time (Q5) } \\
\hline Independent variables & $\begin{array}{l}\text { Odds } \\
\text { ratio }\end{array}$ & $\begin{array}{l}95 \% \text { confidence } \\
\text { interval }\end{array}$ & $\begin{array}{c}P \\
\text { value }\end{array}$ & Independent variables & $\begin{array}{l}\text { Odds } \\
\text { ratio }\end{array}$ & $\begin{array}{l}95 \% \text { confidence } \\
\text { interval }\end{array}$ & $\begin{array}{c}P \\
\text { value }\end{array}$ \\
\hline Patient characteristics & & & & Left main trunk lesion & 2.802 & $1.467-5.353$ & 0.002 \\
\hline Age & 1.014 & $0.989-1.039$ & 0.281 & Left main trunk bifurcation & 2.883 & $1.249-6.654$ & 0.013 \\
\hline Male sex & 1.012 & $0.565-1.814$ & 0.968 & lesion & & & \\
\hline Height (per $10-\mathrm{cm}$ incre- & 0.898 & $0.725-1.112$ & 0.324 & Ostia lesion & 1.421 & $0.682-2.963$ & 0.348 \\
\hline ment) & & & & Bifurcation lesion & 2.145 & $1.238-3.717$ & 0.006 \\
\hline $\begin{array}{l}\text { Body weight (per } 10-\mathrm{kg} \text { in- } \\
\text { crement) }\end{array}$ & 0.892 & $0.731-1.090$ & 0.264 & $\begin{array}{l}\text { Initial TIMI flow grade of } \\
\text { culprit }\end{array}$ & & & \\
\hline Systolic blood pressure & 1.019 & $0.937-1.107$ & 0.660 & 3 & 1.523 & $0.904-2.567$ & 0.114 \\
\hline $\begin{array}{l}\text { (mmHg) (per } 10-\mathrm{mmHg} \text { in- } \\
\text { crement) }\end{array}$ & & & & $\begin{array}{l}\text { Versus 0-2 } \\
\text { Lesion length (mm) (per }\end{array}$ & 1.148 & $0.942-1.399$ & 0.173 \\
\hline Diastolic blood pressure & 0.908 & $0.795-1.038$ & 0.157 & $10-\mathrm{mm}$ increment) & & & \\
\hline $\begin{array}{l}\text { (mmHg) (per } 10-\mathrm{mmHg} \text { in- } \\
\text { crement) }\end{array}$ & & & & $\begin{array}{l}\text { Reference diameter }(\mathrm{mm}) \\
\text { Tortuosity }\end{array}$ & 1.032 & $0.711-1.498$ & 0.870 \\
\hline $\begin{array}{l}\text { Heart rate (beat per minute) } \\
\text { (per } 10 \text {-bpm increment) } \\
\text { Underlying disease }\end{array}$ & 0.942 & $0.834-1.064$ & 0.337 & $\begin{array}{l}\text { Excessive tortuosity } \\
\text { Versus Mild to moderate } \\
\text { tortuosity }\end{array}$ & 3.831 & $1.247-11.773$ & 0.019 \\
\hline Hypertension & 1.679 & $0.683-4.129$ & 0.259 & Eccentricity & & & \\
\hline Diabetes mellitus & 1.222 & $0.729-2.047$ & 0.446 & Eccentric & 1.327 & $0.574-3.067$ & 0.508 \\
\hline Dyslipidemia & 1.118 & $0.611-2.043$ & 0.718 & Versus concentric & & & \\
\hline Hemodialysis & 1.343 & $0.692-2.606$ & 0.383 & Calcification & & & \\
\hline History of previous MI & 0.555 & $0.284-1.085$ & 0.085 & Moderate to severe & 6.514 & $3.746-11.326$ & $<0.001$ \\
\hline History of previous PCI & 0.987 & $0.578-1.684$ & 0.960 & Versus none to mild & & & \\
\hline $\begin{array}{l}\text { History of previous } \\
\text { CABG }\end{array}$ & 2.676 & $1.249-5.734$ & 0.011 & $\begin{array}{l}\text { Occurrence of slow flow in } \\
\text { PCI }\end{array}$ & 2.743 & $0.870-8.648$ & 0.085 \\
\hline Current smoker & 0.556 & $0.278-1.113$ & 0.097 & Procedural characteristics & & & \\
\hline $\begin{array}{l}\text { Medication before admis- } \\
\text { sion }\end{array}$ & & & & $\begin{array}{l}\text { Not ad hoc PCI } \\
\text { Approach site }\end{array}$ & 0.902 & $0.527-1.546$ & 0.708 \\
\hline Aspirin & 1.077 & $0.639-1.813$ & 0.781 & Trans radial coronary in- & 0.465 & $0.276-0.783$ & 0.004 \\
\hline Thienopyridine & 1.372 & $0.809-2.325$ & 0.240 & tervention & & & \\
\hline Beta blocker & 1.068 & $0.630-1.810$ & 0.806 & Versus TFI or TBI & & & \\
\hline ACE inhibitor, ARB & 1.029 & $0.613-1.726$ & 0.915 & Change of approach site & 4.171 & $1.628-10.687$ & 0.003 \\
\hline Calcium channel blocker & 1.070 & $0.633-1.810$ & 0.800 & PCI procedure & & & \\
\hline Statin & 1.156 & $0.685-1.950$ & 0.587 & Drug-eluting stent & 1.177 & $0.630-2.197$ & 0.609 \\
\hline Diuretic & 0.521 & $0.246-1.104$ & 0.089 & Versus Others & & & \\
\hline Hypoglycemic agents & 1.303 & $0.754-2.251$ & 0.343 & Size of guiding catheter & & & \\
\hline Insulin & 0.564 & $0.192-1.661$ & 0.299 & $\geq 7$ & 3.174 & $1.822-5.529$ & $<0.001$ \\
\hline $\begin{array}{l}\text { Laboratory data at admis- } \\
\text { sion }\end{array}$ & & & & $\begin{array}{l}\text { Versus } 6 \mathrm{Fr} \\
\text { Number of guiding catheter }\end{array}$ & & & \\
\hline Serum creatinine $(\mathrm{mg} / \mathrm{dL})$ & 1.035 & $0.952-1.125$ & 0.419 & $\geq 2$ & 4.704 & $2.201-10.054$ & $<0.001$ \\
\hline $\begin{array}{l}\text { Estimated GFR (mL/min- } \\
\left.\text { ute } / 1.73 \mathrm{~m}^{2}\right)\end{array}$ & 0.999 & $0.990-1.007$ & 0.742 & $\begin{array}{l}\text { Versus } 1 \\
\text { Number of wire }\end{array}$ & & & \\
\hline Hemoglobin $(\mathrm{g} / \mathrm{dL})$ & 0.973 & $0.864-1.095$ & 0.650 & $\geq 2$ & 5.458 & $2.990-9.962$ & $<0.001$ \\
\hline BNP $(p g / m L) \quad($ per 100 & 0.985 & $0.949-1.021$ & 0.412 & Versus 1 & & & \\
\hline $\begin{array}{l}\mathrm{pg} / \mathrm{mL} \text { increment) } \\
\text { Killip class }\end{array}$ & & & & $\begin{array}{l}\text { Number of stent } \\
\geq 2\end{array}$ & 4.455 & $2.295-8.647$ & $<0.001$ \\
\hline $3-4$ & 0.744 & $0.377-1.466$ & 0.392 & Versus $0-1$ & & & \\
\hline Versus 1-2 & & & & Number of balloon & & & \\
\hline $\begin{array}{l}\text { Cardiac arrest at prehospi- } \\
\text { tal or ER }\end{array}$ & 0.635 & $0.140-2.879$ & 0.556 & $\begin{array}{l}\geq 3 \\
\text { Versus } 0-2\end{array}$ & 6.064 & $3.472-10.590$ & $<0.001$ \\
\hline $\begin{array}{l}\text { Shock vital at prehospital } \\
\text { or ER }\end{array}$ & 1.125 & $0.440-2.876$ & 0.806 & $\begin{array}{l}\text { Use of extension guide } \\
\text { catheter }\end{array}$ & 3.245 & $1.724-6.109$ & $<0.001$ \\
\hline Lesion characteristics & & & & Use of microcatheter & 6.758 & $3.842-11.888$ & $<0.001$ \\
\hline Culprit lesion & & & & Use of aspiration catheter & 1.265 & $0.339-4.720$ & 0.726 \\
\hline $\begin{array}{l}\text { Left main - left anterior } \\
\text { descending artery }\end{array}$ & 0.723 & $0.426-1.225$ & 0.081 & $\begin{array}{l}\text { Use of rotational coronary } \\
\text { atherectomy }\end{array}$ & 0.190 & $0.093-0.389$ & $<0.001$ \\
\hline Versus others & & & & Temporary pacemaker & 7.048 & $2.422-20.509$ & $<0.001$ \\
\hline $\begin{array}{l}\text { Number of narrowed coro- } \\
\text { nary arteries }\end{array}$ & & & & $\begin{array}{l}\text { Intra-aortic balloon pump- } \\
\text { ing support }\end{array}$ & 3.464 & $1.610-7.456$ & 0.001 \\
\hline Three-vessel disease & 2.241 & $1.309-3.835$ & 0.003 & V-A ECMO & 8.824 & $1.584-49.161$ & 0.013 \\
\hline
\end{tabular}


Table III. Multivariate Logistic Regression Analysis Model to Find Association with Long Fluoroscopy Time

\begin{tabular}{lccr}
\hline \multicolumn{2}{c}{ Dependent variable: long fluoroscopy time (Q5) } & & \\
Independent variables & Odds ratio & 95\% confidence interval & $P$ value \\
\hline Previous CABG & 3.291 & $1.374-7.881$ & 0.008 \\
Bifurcation lesion & 2.516 & $1.356-4.667$ & 0.003 \\
Excessive tortuosity (versus mild to moderate tortuosity) & 4.123 & $1.156-14.699$ & 0.029 \\
Moderate to severe calcification (versus none to mild) & 6.268 & $3.536-11.111$ & $<0.001$ \\
\hline
\end{tabular}

The stepwise model includes the following variables: history of previous CABG, number of narrowed coronary arteries, left main trunk lesion, bifurcation lesion, tortuosity, calcification, approach site, and use of rotational atherectomy.

the difficulties in PCI to the culprit lesions of NSTEMI, which would be helpful for junior PCI operators to ask support of senior PCI operators. Moreover, if there were multiple factors, such as severe tortuosity or calcification in the culprit lesion of NSTEMI, we may consider CABG or optimal medical therapy as an alternative of PCI, especially when patients have multivessel disease. Furthermore, interventional cardiologists should try to reduce fluoroscopy time to prevent health problems caused by radiation exposure. The International Commission on Radiological Protection (ICRP) provided revised recommendation on radiation protection in cardiology. ${ }^{35)}$ Our results may be helpful for operators to recognize which lesions would be associated with more fluoroscopy time in the context of radiation protection.

Study Limitations: First, because this study was a singlecenter, retrospective, observational study, there is a risk of patient selection bias. Second, we arbitrary defined long fluoroscopy time as the longest quintile of the fluoroscopy time, because there were no established cutoff points for long fluoroscopy time. The above methods were sometimes used in early literatures. ${ }^{36-38)}$ Third, because the fluoroscopy time included the time for coronary angiography, as well as the time for PCI, some patients might consume a long fluoroscopy time for coronary angiography, especially in patients with CABG. We could not separate the fluoroscopy time for coronary angiography from the total fluoroscopy time in the present study. Fourth, long fluoroscopy time may be related to the experience of each operator, which could not be adjusted in the present study.

\section{Conclusions}

In PCI to the culprit lesion of NSTEMI, previous CABG, bifurcation, excessive tortuosity, and moderate to severe calcification were associated with long fluoroscopy time. Our study provided a reference for PCI operators to identify the difficulties in PCI to the culprit lesions of NSTEMI.

\section{Acknowledgments}

The authors acknowledge all staff in the catheter laboratory, ICU/CCU, and cardiology ward in Saitama Medical Center, Jichi Medical University, for their technical support in this study.

\section{Disclosure}

Conflicts of interest: Dr. Sakakura has received speaking honoraria from Abbott Vascular, Boston Scientific, Kaneka, Medtronic Cardiovascular, Terumo, OrbusNeich, Japan Lifeline, and NIPRO. He has served as a proctor for Rotablator for Boston Scientific and has served as a consultant for Abbott Vascular and Boston Scientific. Prof. Fujita served as a consultant for Mehergen Group Holdings, Inc.

\section{References}

1. Tunstall-Pedoe H, Kuulasmaa K, Amouyel P, Arveiler D, Rajakangas AM, Pajak A. Myocardial infarction and coronary deaths in the World Health Organization MONICA Project. Registration procedures, event rates, and case-fatality rates in 38 populations from 21 countries in four continents. Circulation 1994; 90: 583-612.

2. Ibanez B, James S, Agewall S, et al. 2017 ESC Guidelines for the management of acute myocardial infarction in patients presenting with ST-segment elevation: The Task Force for the management of acute myocardial infarction in patients presenting with ST-segment elevation of the European Society of Cardiology (ESC). Eur Heart J 2018; 39: 119-77.

3. Roffi M, Patrono C, Collet JP, et al. 2015 ESC Guidelines for the management of acute coronary syndromes in patients presenting without persistent ST-segment elevation: Task Force for the Management of Acute Coronary Syndromes in Patients Presenting without Persistent ST-Segment Elevation of the European Society of Cardiology (ESC). Eur Heart J 2016; 37: 267315 .

4. Goldberg RJ, Currie K, White K, et al. Six-month outcomes in a multinational registry of patients hospitalized with an acute coronary syndrome (the Global Registry of Acute Coronary Events [GRACE]). Am J Cardiol 2004; 93: 288-93.

5. Terkelsen CJ, Lassen JF, Nørgaard BL, et al. Mortality rates in patients with ST-elevation vs. non-ST-elevation acute myocardial infarction: Observations from an unselected cohort. Eur Heart J 2005; 26: 18-26.

6. Ishihara M, Fujino M, Ogawa $\mathrm{H}$, et al. Clinical presentation, management and outcome of Japanese patients with acute myocardial infarction in the troponin era - Japanese Registry of Acute Myocardial Infarction Diagnosed by universal definition (J-MINUET). Circ J 2015; 79: 1255-62.

7. Lee MS, Sillano D, Latib A, et al. Multicenter international registry of unprotected left main coronary artery percutaneous coronary intervention with drug-eluting stents in patients with myocardial infarction. Catheter Cardiovasc Interv 2009; 73: 1521.

8. Kimura K, Kimura T, Ishihara M, et al. JCS 2018 Guideline on Diagnosis and Treatment of Acute Coronary Syndrome. Circ J 2019; 83: 1085-196.

9. Ragmin F, Fast Revascularisation during InStability in Coronary artery disease (FRISC II) Investigators. Invasive compared with 
non-invasive treatment in unstable coronary-artery disease: FRISC II prospective randomised multicentre study. Fragmin and Fast revascularisation during InStability in coronary artery disease Investigators. Lancet 1999; 354: 708-15.

10. Suzuki Y, Tsuchikane E, Katoh O, et al. Outcomes of percutaneous coronary interventions for chronic total occlusion performed by highly experienced Japanese specialists: The first report from the Japanese CTO-PCI Expert Registry. JACC Cardiovasc Interv 2017; 10: 2144-54.

11. Levi A, Kornowski R, Vaduganathan M, et al. Incidence, predictors, and outcomes of failed primary percutaneous coronary intervention: A 10-year contemporary experience. Coron Artery Dis 2014; 25: 145-51.

12. Sakakura K, Ako J, Wada H, Kubo N, Momomura S. ACC/AHA classification of coronary lesions reflects medical resource use in current percutaneous coronary interventions. Catheter Cardiovasc Interv 2012; 80: 370-6.

13. Zanca F, Collard C, Alexandre N, et al. Patient exposure data and operator dose in coronary interventional procedures: Impact of body-mass index and procedure complexity. Phys Med 2020; 76: 38-43.

14. Hasegawa H, Sakakura K, Hamamoto K, et al. Determinants of Greater Peak radiation skin dose in contemporary percutaneous coronary interventions. Cardiovasc Revasc Med 2020; 21: 6-11.

15. Alomari I, Seto A. Approach to treatment of bifurcation lesions. Curr Treat Options Cardiovasc Med 2016; 18: 5.

16. Darremont O, Leymarie JL, Lefèvre T, Albiero R, Mortier P, Louvard Y. Technical aspects of the provisional side branch stenting strategy. EuroIntervention 2015; 11(Suppl V): V86-90.

17. Thygesen K, Alpert JS, Jaffe AS, et al. Fourth universal definition of myocardial infarction (2018). J Am Coll Cardiol 2018; 72: 2231-64.

18. Alpert JS, Thygesen K, Antman E, Bassand JP. Myocardial infarction redefined--A consensus document of the Joint European Society of Cardiology/American College of Cardiology Committee for the redefinition of myocardial infarction. J Am Coll Cardiol 2000; 36: 959-69.

19. Noguchi M, Sakakura K, Akashi N, et al. The comparison of clinical outcomes between inferior ST-elevation myocardial infarction with right ventricular infarction versus without right ventricular infarction. Int Heart J 2019; 60: 560-8.

20. Kubota M, Sakakura K, Yamamoto K, et al. Mid-term clinical outcomes of immediate protamine use following elective percutaneous coronary interventions. Int Heart J 2020; 61: 865-71.

21. Matsuo S, Imai E, Horio M, et al. Revised equations for estimated GFR from serum creatinine in Japan. Am J Kidney Dis 2009; 53: 982-92.

22. Ellis SG, Vandormael MG, Cowley MJ, et al. Coronary morphologic and clinical determinants of procedural outcome with angioplasty for multivessel coronary disease. Implications for patient selection. Multivessel Angioplasty Prognosis Study Group. Circulation 1990; 82: 1193-202.

23. Asada S, Sakakura K, Taniguchi Y, et al. Association of the long fluoroscopy time with factors in contemporary primary percuta- neous coronary interventions. PLoS One 2020; 15: e0237362.

24. Mintz GS, Popma JJ, Pichard AD, et al. Patterns of calcification in coronary artery disease. A statistical analysis of intravascular ultrasound and coronary angiography in 1155 lesions. Circulation 1995; 91: 1959-65.

25. Raza S, Blackstone EH, Houghtaling PL, et al. Natural history of moderate coronary artery stenosis after surgical revascularization. Ann Thorac Surg 2018; 105: 815-21.

26. Kasahara T, Sakakura K, Momomura SI, Fujita H. A modified reverse guidewire technique for a Crusade-uncrossable bifurcation lesion. J Cardiol Cases 2020; 21: 32-4.

27. Shlofmitz E, Khalid N. Calcified plaque. StatPearls. Treasure Island (FL): StatPearls Publishing Copyright(C) 2020. StatPearls Publishing LLC 2020.

28. Nikolsky E, Gruberg L, Pechersky S, et al. Stent deployment failure: Reasons, implications, and short- and long-term outcomes. Catheter Cardiovasc Interv 2003; 59: 324-8.

29. Wilson SJ, Maeremans J, Walsh SJ, et al. The first clinical experience with a novel "locking" microcatheter in chronic coronary total occlusions. EuroIntervention 2017; 12: e1883-8.

30. Gorol J, Tajstra M, Hudzik B, Lekston A, Gassior M. Comparison of outcomes in patients undergoing rotational atherectomy after unsuccessful coronary angioplasty versus elective rotational atherectomy. Postepy Kardiol Interwencyjnej 2018; 14: 128-34.

31. Kassimis G, Raina T, Kontogiannis N, et al. How should we treat heavily calcified coronary artery disease in contemporary practice? From atherectomy to intravascular lithotripsy. Cardiovasc Revasc Med 2019; 20: 1172-83.

32. Hirai K, Sakakura K, Watanabe Y, et al. Determinants of high device cost in current percutaneous coronary interventions. Cardiovasc Revasc Med 2018; 19: 607-12.

33. Daida H, Miyauchi K, Ogawa H, et al. Management and twoyear long-term clinical outcome of acute coronary syndrome in Japan: Prevention of atherothrombotic incidents following ischemic coronary attack (PACIFIC) registry. Circ J 2013; 77: 934-43.

34. Lange RA, Hillis LD. Should thrombolysis or primary angioplasty be the treatment of choice for acute myocardial infarction? Thrombolysis--the preferred treatment. N Engl J Med 1996; 335: 1311-2; discussion 6-7.

35. Cousins C, Miller DL, Bernardi G, et al. ICRP PUBLICATION 120: Radiological protection in cardiology. Ann ICRP 2013; 42: $1-125$.

36. Wehinger A, Kastrati A, Elezi S, et al. Lipoprotein(a) and coronary thrombosis and restenosis after stent placement. J Am Coll Cardiol 1999; 33: 1005-12.

37. Groenland CNL, Termorshuizen F, Rietdijk WJR, et al. Emergency Department to ICU time is associated with hospital mortality: A registry analysis of 14,788 patients from six university hospitals in the Netherlands. Crit Care Med 2019; 47: 1564-71.

38. Khera AV, Emdin CA, Drake I, et al. Genetic risk, adherence to a healthy lifestyle, and coronary disease. N Engl J Med 2016; 375: 2349-58. 